\title{
DVO-Kursstaffel 2019
}

Die OSTAK Osteologie Akademie GmbH freut sich, Ihnen die DVO-Kursstaffel 2019 vorstellen zu können. Auch im nächsten Jahr werden wieder Fortbildungen für die Zertifizierung und Rezertifizierung zur Osteologin DVO/zum Osteologen DVO angeboten. Darüber hinaus spielen die neuen Basiskurse Osteologie zum Thema "Allgemeine Osteoporoseversorgung" eine große Rolle: Sie sind nach dem neuen DVO-Curriculum nicht nur eine der Voraussetzungen für die Zertifizierung zur Osteologin DVO/zum Osteologen DVO, sondern auch Bestandteil des ZertifikatsErwerbs für Expertinnen/Experten für allgemeine Osteoporoseversorgung und Expertinnen/Experten für spezielle Osteoporoseversorgung.

\section{Grundkurse}

Den Auftakt für die Kursstaffel 2019 macht Kursleiter Prof. Dr. med. Klaus M. Peters mit dem Grundkurs III zum Thema „Osteologische Erkrankungen jenseits der Osteoporose". Die Veranstaltung findet bereits am 26. +27. Januar 2019 in Essen statt.

Besucher des Kongresses der Deutschen Gesellschaft für Endokrinologie (DGE) haben die Möglichkeit, Ihren Kongressaufenthalt mit einer Teilnahme am Grundkurs II zum Thema „Diagnostik der Osteoporose $^{\text {" }} \mathrm{zu}$ verbinden: Der Kurs findet am 23.+24. März 2019 unter der Leitung von Prof. Dr. rer. nat. Claus-C. Glïer im Anschluss an den DGE-Kongress in Göttingen statt.

\section{Spezialkurse}

Am Mittwoch, dem 27. März 2019, läutet Univ.-Prof. Dr. med. Dr. phil. Peter Herbert Kann M.A. die Kongresstage der OSTEOLOGIE 2019 in Frankfurt am Main mit dem Spezialkurs zum Thema „Hot Topics der Osteologie 2019" ein. Auch die beiden Kongresspräsidenten, Prof. Dr. med. Andreas Kurth und Prof. Dr. med. Peyman Hadji, werden an diesem Kurs mitwirken.
Wissenswertes zum Thema „Der Knochen in der rheumatologischen Praxis" können Interessierte im Spezialkurs am 18. Mai 2019 in Berlin erfahren. „Meine Referenten und ich werden in besonderem Maße die osteologischen Aspekte bei rheumatologischen Erkrankungen und die Auswirkungen von antirheumatischer Medikation auf den Knochen behandeln. Selbstverständlich wird es auch wieder interaktive Falldiskussionen geben.", beschreibt Kursleiter Prof. Dr. med. Frank Buttgereit die Schwerpunkte seiner Veranstaltung.

Auch den beliebten Adventskurs bietet die OSTAK Osteologie Akademie GmbH 2019 wieder an: Die Kursleiter Prof. Dr. med. Christopher Niedhart, Prof. Dr. med. Uwe Maus und Prof. Dr. med. Klaus M. Peters freuen sich darauf, am 07. Dezember 2019 in Köln gemeinsam mit Ihnen beim Spezialkurs zum Thema „Der „schwierige“ Patient - ungelöste Einzelfälle und Hochrisikopatienten " zu diskutieren. „Bei dem Kurs werden wir uns mit besonderen osteologischen Risikosituationen, Nebenwirkungen der medikamentösen Therapie und der Langzeitbehandlung von Osteoporose beschäftigen. Dabei ist uns der Dialog mit unseren Teilnehmern sehr wichtig.", erläutert Professor Niedhart die Intentionen des Kursleiter-Teams.

\section{Basiskurse}

Zukünftige Osteologinnen DVO/Osteologen DVO, Expertinnen/Experten für spezielle Osteoporoseversorgung und Expertinnen/Experten für allgemeine Osteoporoseversorgung haben die Möglichkeit, 2019 aus fünf Basiskursen Osteologie zum Thema „Allgemeine Osteoporoseversorgung“ zu wählen. „Mit diesen Kursen möchten wir auf die steigende Anzahl der Osteoporose-Patienten, die mit dem demografischen Wandel einhergeht, vorbereiten: Die Volkskrankheit wird zukünftig in fast allen medizinischen Fachdisziplinen eine Rolle spielen. Der Basiskurs Osteologie und die nachfolgende weitere Qualifizierung soll einen fundierten Einblick in die Diagnostik und Therapie des Krankheits- bildes geben und die Kollegen dazu befähigen, die betroffenen Patienten evidenzbasiert und kompetent zu beraten und zu behandeln.", beschreibt Prof. Dr. med. Andreas Kurth, erster Vorsitzender des Dachverbandes Osteologie e.V. (DVO), Ziel und Inhalt der neuen Basiskurse.

Der erste Basiskurs Osteologie der neuen Kursstaffel findet am 30.+31. März 2019 unter der Leitung von Univ.-Prof. Dr. med. Uwe Lange in Frankfurt am Main direkt im Anschluss an die OSTEOLOGIE 2019 statt. Kongressaufenthalt und Kursbesuch können auch hier miteinander verbunden werden. Diese Idee greift der DVO erneut bei den Basiskursen am 02.+03. Mai 2019 in Wiesbaden und am 04.+05. Mai 2019 in Baden-Baden auf: Ersterer findet unter der Leitung von Prof. Dr. med. Franz Jakob im Rahmen des Kongresses der Deutschen Gesellschaft für Innere Medizin e.V. (DGIM) statt, während Kursleiter Dr. med. Hermann Schwarz seine Teilnehmer direkt im Anschluss an den Kongress der Vereinigung Süddeutscher Orthopäden und Unfallchirurgen e.V. (VSOU) begrüßt.

Auch in Nordrhein-Westfalen und im "hohen Norden" veranstaltet die OSTAK Osteologie Akademie GmbH Basiskurse Osteologie: Dr. med. Christian Wittig, Kursleiter und Sprecher der Osteologischen Zentrenleiter DVO, empfängt alle Interessierten am 14. +15. September 2019 in Düsseldorf, während Prof. Dr. med. Florian Barvencik sich auf seine Teilnehmerinnen und Teilnehmer am 16.+17. November 2019 in Hamburg freut.

\section{Anmeldung}

Wir hoffen, wir konnten Sie neugierig auf die neue Kursreihe machen und würden uns freuen, Sie im nächsten Jahr bei einer unserer Veranstaltungen begrüßen zu dürfen. Traditionell besteht wieder die Möglichkeit, sich ab dem 01 . Oktober 2018 online auf www.ostak.de zu den Kursen anzumelden.

Katharina Kirchner, OSTAK Osteologie Akademie GmbH 


\section{Grundkurse 2019}

\section{6. +27. Januar 2019}

Grundkurs III: Osteologische Erkrankungen jenseits der Osteoporose Ort: Essen

Leitung: Prof. Dr. med. Klaus M. Peters

\section{3. + 24. März 2019}

Grundkurs II: Diagnostik der Osteoporose

Ort: Göttingen

Leitung: Prof. Dr. rer. nat. Claus-C. Glüer Kurs schließt an den Kongress der DGE an.

\section{Spezialkurse 2019}

\section{März 2019}

Hot Topics der Osteologie 2019

Ort: Frankfurt am Main

Leitung: Univ.-Prof. Dr. med. Dr. phil. Peter

Herbert Kann M.A.

Kongress OSTEOLOGIE 2019 beginnt am nächsten Tag.

\section{Mai 2019}

Der Knochen in der rheumatologischen

\section{Praxis}

Ort: Berlin

Leitung: Prof. Dr. med. Frank Buttgereit

\section{Dezember 2019}

Der „schwierige" Patient - ungelöste Einzelfälle und Hochrisikopatienten Ort: Köln

Leitung: Prof. Dr. med. Christopher Niedhart, Prof. Dr. med. Uwe Maus, Prof. Dr. med. Klaus M. Peters

Save the Date: 28. - 30.03.2019

\section{OSTEOLOGIE 2019}

Tagungsort: Kap Europa, Osloer Str. 5, Abstracteinreichung: bis 01.10.2018 D-60327 Frankfurt am Main

Kongresspräsidenten: Prof. Dr. med. Peyman Hadji (Frankfurt am Main), Prof. Dr. med. Andreas Kurth (Mainz)

Motto: Healthy Aging - Bone and Joint

\section{Schwerpunktthemen:}

- „Neues und Bewährtes" in der Diagnostik und der Therapie von Knochenerkrankungen

- Osteoonkologie

Kinderosteologie

Alterstraumatologie

Wirbelsäulenerkrankungen

Arthrose

Knochenersatzmaterialien

Östrogeneffekte auf den Knochen

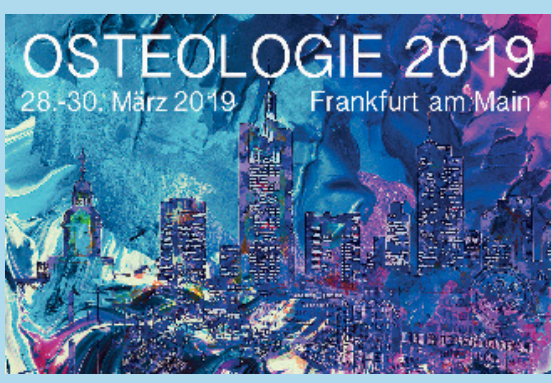

Early-bird-Anmeldung: 01.10.-30.11.2018

Weitere Informationen:

www.osteologie2019.de

Stand der Informationen: August 2018. Änderungen vorbehalten.

\section{Basiskurse 2019}

\section{0. + 31. März 2019}

Allgemeine Osteoporoseversorgung

Ort: Frankfurt am Main

Leitung: Univ.-Prof. Dr. med. Uwe Lange Kurs schließt an die OSTEOLOGIE 2019 an.

02. +03. Mai 2019

Allgemeine Osteoporoseversorgung Ort: Wiesbaden

Leitung: Prof. Dr. med. Franz Jakob

Der Kongress der DGIM beginnt am nächsten Tag.

04. + 05. Mai 2019

Allgemeine Osteoporoseversorgung Ort: Baden-Baden

Leitung: Dr. med. Hermann Schwarz Kurs schließt an den Kongress der VSOU an.

14. + 15. September 2019

Allgemeine Osteoporoseversorgung Ort: Düsseldorf

Leitung: Dr. med. Christian Wittig

16. +17 . November 2019

Allgemeine Osteoporoseversorgung Ort: Hamburg Leitung: Prof. Dr. med. Florian Barvencik

\section{Impressum}

Verantwortlich für den Inhalt

Yvonne Bodden, Essen 